\title{
Unidad Médica de Corta Estancia
}

En el presente número de Anales de Medicina Interna se describe la eficiencia de una Unidad de Corta Estancia por el análisis del reingreso hospitalario no planificado (1).

La limitación en la disponibilidad de camas para hospitalización y el intento de contener el incremento del gasto sanitario han contribuido a la búsqueda de alternativas a la hospitalización convencional. En esta línea puede encuadrarse el desarrollo de hospitales de día, unidades de observación, hospitalización a domicilio o las unidades de corta estancia.

La sanidad en España constituye por su cuantía el segundo programa de protección social, absorbiendo casi el 7\% del Producto Interior Bruto. Además de factores como el demográfico, envejecimiento de la población, tasa de cobertura o el gasto en nuevas tecnologías, influye en ese gasto la evolución de las prácticas médicas. Parámetros en los que éstas se reflejan son el personal sanitario por cama, utilización cama/día, uso de servicios médicos o el consumo de farmacia (2).

Al identificarse la duración de la estancia como uno de los principales determinantes del coste de una hospitalización, se ha desarrollado como una estrategia prioritaria para la mejora de la eficiencia, reducir la estancia hospitalaria de los pacientes manteniendo los niveles de calidad (3).

La idoneidad de la estancia hospitalaria en cada patología y en cada enfermo es sin duda difícil de establecer. Entre los instrumentos para su medida que más se han extendido se encuentra la aplicación del "Appropriateness Evaluation Protocol" (4). En su versión original incluía 27 parámetros, tanto de los cuidados que se administran, como de la situación del paciente. Cuando no se cumple ninguno de ellos, se considera inadecuado ese día de hospitalización. Se han realizado diversas adaptaciones de estos criterios en distintos países (5-11). Todos los estudios realizados con esta metodología en numerosos países y con diferentes modelos sanitarios, coinciden en apreciar un número significativo de estancias hospitalarias inadecuadas (29-45\%). A estos datos no se les puede conceder una validez absoluta, pues existen factores difícilmente estandarizables que pueden no ser detectados por la aplicación de un protocolo (12). No obstante aportan una información valorable que si se añade a la conocida variabilidad de un centro a otro para un mismo proceso (13), nos lleva a considerar que una mayor eficiencia en los procesos per- mitiría un acortamiento en la duración de las hospitalizaciones sin deterioro de la calidad asistencial.

Las Unidades Médicas de Corta Estancia nacen con la hipótesis de que la eficiencia y calidad de la atención a un paciente que no requiere una estancia hospitalaria prolongada, puede mejorar si es ingresado en una unidad diferenciada y con médicos familiarizados en la atención a pacientes de esas características, que no deban simultanear su atención con la de pacientes más complejos.

Entre los factores para el correcto funcionamiento de estas unidades, se ha destacado la importancia de una adecuada selección de los pacientes, que debería ser realizada por el mismo equipo que atiende la unidad; no utilizar la unidad para pacientes sin las características prefijadas, para lo que es fundamental un número de camas adecuado a las características del centro, y nunca superior; y tener organizado un sistema eficaz de seguimiento en consulta externa (14).

Son escasas las publicaciones que analizan el funcionamiento de estas unidades en los departamentos de Medicina Interna. Bazarian y cols. (15), y Abenhaim HA y cols. (14), han comunicado su experiencia en sendas unidades de 16 camas llevada por internistas con una estancia media de 2,4 días. Los diagnósticos más habituales fueron: asma y enfermedad pulmonar obstructiva crónica, neumonía, insuficiencia cardiaca, infección urinaria y celulitis. El $80 \%$ de los pacientes hospitalizados en la unidad fueron dados de alta desde la misma, precisando traslado a otras el $20 \%$, siendo la mortalidad inferior al $1 \%$. Fueron reingresados el $2 \%$ en los tres días siguientes al alta, $1,2 \%$ en el período de cuatro días a una semana y el $6,1 \%$ entre una semana y un mes, totalizando por tanto los reingresos hospitalarios a los 30 días el 9,6\% (14).

En nuestro país también se ha comunicado la experiencia con estas unidades (16-18). En la literatura hay descripciones de Unidades de Corta Estancia en unidades pediátricas (19), departamentos de Urgencias (20), o para procesos específicos como traumatismos craneales, intoxicaciones, o para descartar síndromes coronarios agudos en pacientes de bajo riesgo (21).

El índice de rehospitalizaciones suele aceptarse como uno de los parámetros a controlar para valorar la calidad de la asistencia hospitalaria, especialmente cuanto más precoz se pro- 
duce (22). No obstante no se ha demostrado de forma consistente la asociación entre reingresos y una atención de baja calidad (23). La falta de uniformidad en los criterios limitan el valor de este dato (24).

Entre el 9 y el $48 \%$ de los reingresos están asociados con indicadores de una atención no óptima, como escasa resolución del problema que motivó el ingreso, tratamiento de alta impreciso, o una atención extrahospitalaria inadecuada (25). Probablemente su valor como indicador sea mayor cuando se aplica a pacientes con patologías concretas, (diabetes, asma, bronquitis crónica), en los que índices elevados de rehospitalización pueden permitir detectar deficiencias en la calidad de la atención (26). Los reingresos que se producen en períodos más tardíos de la hospitalización índice, estarán relacionados fundamentalmente con el estado general del paciente y la progresión de enfermedades crónicas, perdiendo por tanto utilidad como indicador de calidad hospitalaria, pero si expresa la necesidad de programas de atención específica a estos pacientes (25).

\section{Bibliografía}

1. Diz-Lois MT, De la Iglesia F, Nicolás R, Pellicer C, Ramos V, Diz-Lois F. Factores predictores de reingreso hospitalario no planificado en pacientes dados de alta de una Unidad de Corta Estancia Médica. An Med Interna (Madrid) 2002; 19: 221-225.

2. Barea J. Organización hospitalaria y eficiencia. Gestión y Evaluación de Costes Sanitarios 2001; 2: 5-11.

3. Clarke A. Why are we trying to reduce lenght of stay?. Evaluation of the costs and benefits of reducing time in hospital must start from the objetives that govern change. Quality in Health Care 1996; 5: 172-9.

4. Gertman PM, Restuccia JD. The Appropriateness evaluation protocol: a technique for assessing unnecessary days of hospital care. Med Care 1981; 19: 855-71.

5. Muiño A, Ramallo VG, Rodriguez de Castro E, Lázaro C, Fdez-Basave E. Asistencia en un Servicio de Urgencias: Justificación de las visitas y adecuación de los ingresos. Rev Clin Esp 1988; 182: 374-8.

6. Schneeweiss S, Sangha O, Siebert H, Hebeisen H, Hubner M, Heinrich R, Wildner M, Witte J. Evaluation of the appropriateness of hospital care in internal medicine. Reliability of a German adaptation of the procedure. Dtsch Med Wochenschr 2000; 125: 894-9.

7. Bare ML, Prat A, Lledo L, Asenjo MA, Salleras L. Appropriateness of admissions and hospitalization days in an acute-care teaching hospital. Rev Epidemiol Sante Publique 1995; 443: 328-36.

8. Lang T, Liberati A, Tampieri A, Felling G, Gonsalves L, Lorenzo S, et al. A European version of the Appropriateness Evaluation Protocol. Goals and presentation. The BIOMED I Group on Appropriateness of Hospital Use. Int J Technol Asses Health Care 1999; 15: 185-97.

9. Butler JS, Barrett BJ, Kent G, Haire R, Parfrey PS. Detection and classification of inappropriate hospital stay. Clin Invest Med 1996; 19: 251-8.

10. Smith HE, Pryce A, Carlisle L, Jones JM, Scarpello J, Pantin C. Appropriateness of acute medical admissions and length of stay. J R Coll Physicians Lond 1997; 31: 527-32.

11. Angelillo IF, Ricciardi G, Nante N, Boccia A, Bianco A, La Torre G, et al. Appropriateness of hospital utilisation in Italy. Public Health 2000; 114: 9-14.

12. Smeets PM, Verheggen FW, Pop P, Panis W, Carpay JJ. Assessing the necessity of hospital stay by means of the appropriateness evaluation protocol: how strong is the evidence to proceed?. Int J Qual Heath Care 2000; 12: 483-93.

13. Sorasdekkan H. Efficiency analysis of university hospitals. Tiddsskr Nor Laegeforen 1991; 111: 65-70.
El estudio de Diz-Lois y colaboradores (1), reafirma la utilidad de las Unidades de Corta Estancia presentando unas tasas de reingreso en el rango referido en la literatura, originado además en factores no modificables, como es el número de ingresos previos, y no en aspectos relacionados con el método de trabajo de la unidad.

Es necesario que nuevos estudios nos permitan comparar los resultados de estas unidades con los de servicios convencionales en casos de similar complejidad, pero parece muy probable que las Unidades de Corta Estancia favorezcan la eficiencia del uso de las camas hospitalarias con resultados satisfactorios para los pacientes.

\section{A. MUIÑO MIGUEZ}

Hospital Universitario Gregorio Marañón. Madrid
14. Abenhaim HA, Kahn SR, Raffoul J, Becker R. Program description: A hospitalistrun, medical short-stay unit in a teaching hospital. CMAJ 2000; 163: 1477-83.

15. Bazarian JJ, Schneider SM, Newman VJ, Chodosh J. Do admitted patients held in the emergency department impact the throughput of treat-and-release patients?. Acad Emerg Med 1996; 3: 1113-8.

16. De la Iglesia F, Ramos V, Pellicer C, Nicolás R, Diz-Lois F. La Unidad de Corta Estancia Médica de A Coruña: cumplimos cinco años. An Med Interna (Madrid) 2001; 18: 166.

17. De la Iglesia F, Pellicer C, Ramos V, Castro B, Rodríguez A, Diz-Lois F. Unidad de Corta Estancia Médica de La Coruña: nuestra experiencia. An Med Interna (Madrid) 1997; 14: 125-7.

18. Barbado MJ, Jimeno A, Ostolaza JM, Molinero J. Unidad de Corta Estancia dependiente de Medicina Interna. An Med Interna (Madrid) 1999; 16: 504-10.

19. McConnochie KM, Russo MJ, McBride T, Szilagyi PG, Brooks AM, Roghmann KJ. How commonly are children hospitalized for asthma eligible for care in alternative settings?. Arch Pediatr Adolesc Med 1999; 153: $49-55$

20. Muiño A, Gil J, Gabarró N, Segado A, López C. Unidad de observación y corta estancia de medicina en el servicio de urgencias. An Med Interna (Madrid) 1998; 15: 138-41.

21. Gaspoz JM, Lee TH, Weinstein MC, Cook EF, Goldman P, Komaroff AL. Costeffectiveness of a new short-stay unit to "rule out" acute myocardial infartion in low risk patients. J Am Coll Cardiol 1994; 24: 1249-59.

22. Kossovsky MP, Perneger TV, Sarasin FP. Bolla F, Borst F, Gaspoz JM. Comparison between planned and unplanned readmissions to a department of internal medicine. J Clin Epidemiol 1999; 52:151-156.

23. Weinstein J. Hospital readmissions and quality of care. Medical Care 1999; 37: 490-501.

24. Hughes MR, Johnson N J, Nemeth LS. Clasifying reasons for hospital readmissions. Topics in Health Information Management 2000; 20: 65-71.

25. Benbassat J, Taragin M. Hospital readmissions as a measure of quality of heath care: advantages and limitations. Arch Inter Med 2000; 160: 1074-81.

26. Cotton MM, Bucknall CE, Dagg KD, Johnson et al. Early discharge for patients with exacerbations of chronic obstructive pulmonary disease: A randomised controlled trial. Thorax 2000; 55: 902-8. 